\title{
The premiss-based approach to judgment aggregation
}

Citation for published version (APA):

Dietrich, F. K., \& Mongin, P. (2008). The premiss-based approach to judgment aggregation. METEOR, Maastricht University School of Business and Economics. METEOR Research Memorandum No. 013 https://doi.org/10.26481/umamet.2008013

Document status and date:

Published: 01/01/2008

DOI:

10.26481/umamet.2008013

Document Version:

Publisher's PDF, also known as Version of record

\section{Please check the document version of this publication:}

- A submitted manuscript is the version of the article upon submission and before peer-review. There can be important differences between the submitted version and the official published version of record.

People interested in the research are advised to contact the author for the final version of the publication, or visit the DOI to the publisher's website.

- The final author version and the galley proof are versions of the publication after peer review.

- The final published version features the final layout of the paper including the volume, issue and page numbers.

Link to publication

\footnotetext{
General rights rights.

- You may freely distribute the URL identifying the publication in the public portal. please follow below link for the End User Agreement:

www.umlib.nl/taverne-license

Take down policy

If you believe that this document breaches copyright please contact us at:

repository@maastrichtuniversity.nl

providing details and we will investigate your claim.
}

Copyright and moral rights for the publications made accessible in the public portal are retained by the authors and/or other copyright owners and it is a condition of accessing publications that users recognise and abide by the legal requirements associated with these

- Users may download and print one copy of any publication from the public portal for the purpose of private study or research.

- You may not further distribute the material or use it for any profit-making activity or commercial gain

If the publication is distributed under the terms of Article $25 \mathrm{fa}$ of the Dutch Copyright Act, indicated by the "Taverne" license above, 
Franz Dietrich, Philippe Mongin

The Premiss-Based Approach to Judgment

Aggregation

$\mathrm{RM} / 08 / 013$

JEL code: D7

\section{METE@R}

Maastricht research school of Economics

of TEchnology and ORganizations

Universiteit Maastricht

Faculty of Economics and Business Administration P.O. Box 616

NL - 6200 MD Maastricht

phone : ++31433883830

fax : $\quad++31433884873$ 


\title{
The Premiss-Based Approach to Judgment Aggregation
}

\author{
Franz Dietrich \& Philippe Mongin \\ December 2007
}

\begin{abstract}
We investigate judgment aggregation by assuming that some formulas of the agenda are singled out as premisses, and the Independence condition (formula-wise aggregation) holds for them, though perhaps not for others. Whether premiss-based aggregation thus defined is non-degenerate depends on how premisses are logically connected, both among themselves and with other formulas. We identify necessary and sufficient conditions for dictatorship or oligarchy on the premisses, and investigate when these results extend to the whole agenda. Our theorems recover or strengthen several existing ones and are formulated for infinite populations, an innovation of this paper. JEL identification numbers: D70, D71.
\end{abstract}

Keywords: Premiss-based aggregation, judgment aggregation, impossibility theorems, infinite populations, majority voting

\section{Introduction}

Imagine that a group of individuals needs to form collective ("yes" or "no") judgments on certain propositions, e.g., that smoking is harmful $(a)$, smoking should be banned in public places $(b)$, if smoking is harmful, then it should be banned in public places $(a \rightarrow b)$, and so on. Assume further that the collective judgments are obtained by aggregating the individual judgments - this is the judgment aggregation problem. A natural approach consists in singling out those propositions - to be called premisses - which are in some sense basic to the other judgments, aggregating them in some way, and then see what eventually results for the others. We will assume that if a formula $p$ represents a premiss, a decision on $p$ is made independently of all other formulas, for instance, but not necessarily, by majority voting. By contrast, if $\varphi$ represents a non-premiss, the decision on $\varphi$ will typically take into account those made on other formulas; for instance, though again not necessarily, $\varphi$ or its negate $\neg \varphi$ logically follows from the accepted premisses. In the smoking case, if only $a$ and $a \rightarrow b$ count as premisses, they are subjected, say, to majority vote, while $b$ follows or does not follow, depending on the result of the poll. We call such an aggregation procedure locally independent, because it limits the independence condition of logical aggregation theory to a subset of formulas.

What is called a premiss-based procedure in this theory is not quite definite. When it is discussed at all, it is construed in terms of typically several of the following conditions: (i) premisses are subjected to a majority vote; (ii) they are logically 
independent from each other; (iii) if judgments are made for or against each premiss, they entail judgments for or against each non-premiss; (iv) only one formula is not a premiss; (v) the independence condition holds globally on the agenda. The present aggregation functions, which require neither of these restrictions, can then be viewed as an abstraction of the common meanings given to the premiss-based approach.

We ask whether and when locally independent aggregation functions are suitably well-behaved. Our answers take the form of characterizing how the premisses should be interrelated, both among themselves and with the other propositions. As an innovation in the field, we allow the population to be infinite, and show that neither our, nor the existing impossibility results hold in this case if the possibility criterion is not adapted to the new assumption. Depending on how thoroughly it is reformulated, the negative results may or may not be upheld.

Our research was originally motivated by Mongin's (forthcoming) requirement that the aggregation function be independent on the propositional variables - a special case of local independence. While we share the scepticism expressed in this paper about taking separate votes on composite Boolean expressions - why should the decision on a disjunction $a \vee b$ care only about how the individuals judge $a \vee b$, not about how they judge the "building blocks" $a$ and $b$ ? -, we do not take this as a reason to restrict the independence condition to such a special case as the propositional variables. As the smoking example straightforwardly illustrates, a set of premisses may both exclude some propositional variables and include some Boolean formulas, and be nonetheless perfectly intuitive. When the logic includes non-Boolean connectives, say the conditional logic operator of Dietrich (2007), the case for allowing compound formulas is further strengthened.

Our results are general enough to cover Mongin's theorem as a particular case. They also cover several existing theorems, which correspond to another particular case, in which every formula is a premiss; see, e.g., Dietrich and List (2007, forthcoming), Dokow and Holzman (2005, 2006), Gärdenfors (2006), all connected with Nehring and Puppe's (2002) social-choice-theoretic investigation and inspired by List and Pettit's (2002) original analysis. We actually strengthen these results on different scores, in particular showing how they can or cannot be extended to the novel case of infinite populations. Beside unifying a large chunk of the literature, our framework and results make it possible to address unexplored sets or premisses, i .e, those which consist neither of propositional variables, nor of the whole agenda, and these are the truly salient cases for premiss-based aggregation.

\section{The logic and the aggregative framework}

By assumption, the propositions on which the individuals and society make judgments can be expressed by formulas in some logical language; henceforth, we will treat the latter as being themselves the objects of judgment. As in Dietrich (2007), we do not specify the logical language entirely, and just impose general restrictions on it; in this way, many well-known languages of either classical or non-classical logic will be included without saying. Let $\mathcal{L}$ be the non-empty set of formulas of the language. We may assume that $\mathcal{L}$ is inductively constructed from a given set $\mathcal{P}$ of 
propositional variables (p.v.), which may be finite or infinite, using a finite number of connectives, among which the negation symbol $\neg$; in addition, there may be symbols of other Boolean connectives $(\wedge, \vee, \rightarrow, \leftrightarrow$, standing for "and", "or", "implies", "is equivalent to") as well as non-Boolean ones (e.g., a unary modal operator $O$ standing for "it ought to be that", or a binary modal operator standing $\hookrightarrow$ for "implies in the counterfactual sense"). ${ }^{1}$

A formula is a literal if it is a p.v. or the negate of a p.v., and molecular otherwise. It is Boolean if all connectives it involves are Boolean. The Latin letters $a, b, c \ldots$ will be used for p.v., and the Greek letters $\varphi, \psi, \chi \ldots$ for formulas of any kind.

The logical language comes with a logic, which we capture by means of its notion of consistency; entailment is a derivative notion in the present framework. Thus, by assumption, all subsets $S \subseteq \mathcal{L}$ are classified into consistent or inconsistent ones, and by definition, $S \subseteq \mathcal{L}$ entails a formula $\varphi \in \mathcal{L}$ - formally, $S \vdash \varphi-$ if $S \cup\{\neg \varphi\}$ is inconsistent. A formula $\varphi \in \mathcal{L}$ is a theorem if $\{\neg \varphi\}$ is inconsistent, a contradiction if $\{\varphi\}$ is inconsistent, a contingent formula if both $\{\neg \varphi\}$ and $\{\varphi\}$ are consistent. Any partition of $2^{\mathcal{L}}$ can serve as a primitive distinction between consistent and inconsistent sets, provided it satisfies the following conditions: ${ }^{2}$

(L1) $\emptyset$ is consistent.

(L2) For all $\varphi \in \mathcal{L},\{\varphi, \neg \varphi\}$ is inconsistent.

(L3) For all $S \subseteq \mathcal{L}$ and all $\varphi \in \mathcal{L}$, if both $S \cup\{\varphi\}$ and $S \cup\{\neg \varphi\}$ are inconsistent, so is $S$.

(L4) If $S \subseteq \mathcal{L}$ is consistent, all its subsets are consistent; equivalently, if $S \subseteq \mathcal{L}$ is inconsistent, all its supersets in $\mathcal{L}$ are inconsistent.

(L5) Every consistent $S \subseteq \mathcal{L}$ has a consistent superset $T \subseteq \mathcal{L}$ that contains a member of each pair $\varphi, \neg \varphi \in \mathcal{L}$.

(L6) Every inconsistent set $S \subseteq \mathcal{L}$ has a finite inconsistent subset $S_{0} \subseteq S$.

In fact, (L3) follows from (L4) and (L5), hence need not be assumed separately. The conditions (L1)-(L3) already imply some standard properties of $\vdash$, e.g., that if $S \vdash \varphi$ and $S$ is consistent, so is $S \cup\{\varphi\}$, and that if $S \vdash \varphi$ and $S \vdash \neg \varphi$, then $S$ is inconsistent. With (L4) added, other standard properties follow, in particular, the converse of the last-mentioned implication and the monotonicity of $\vdash$, i.e., the property that if $S \vdash \varphi$, then $S \cup T \vdash \varphi$.

In view of these logical properties, (L5) implies that any consistent $S \subseteq \mathcal{L}$ can be extended to a maximal consistent $T \subseteq \mathcal{L}$. It amounts to taking for granted a result of elementary logic, Lindenbaum's extension theorem, which is proved by means of Zorn's lemma. ${ }^{3}$

Aside from (L4), the most constraining assumption is (L6), which implies the compactness of $\vdash$, i.e., the property that if $S \vdash \varphi$, there exists a finite $S_{0} \subseteq S$ such that $S_{0} \vdash \varphi$. The propositional calculus is compact, but probabilistic logics are normally not, and not all modal logics are. ${ }^{4}$ Accordingly, we will always be careful

\footnotetext{
${ }^{1}$ Dietrich (forthcoming) analyses logical aggregation on agendas with subjunctive implications.

${ }^{2}$ Dietrich's (2007) conditions are equivalent but take $\vdash$ as primitive.

${ }^{3}$ See Chang and Keisler, 1973, p. 26, for a statement and proof of Lindenbaum's theorem.

${ }^{4}$ Heifetz and Mongin's (2001) system is an example, among many, of non-compactness in probabilistic logic. While the most elementary modal logics are compact (see Chellas, 1980), those developed
} 
to say where (L6) occurs in our proofs, whereas we will use (L1)-(L5) and their many implications without saying. The issue of compactness arises only if the relevant set of formulas (i.e., the agenda as defined below) is infinite, but this is a case that we take to be relevant to logical aggregation.

When we say below that a logic is propositional, we mean that it includes the standard axiomatization of the Boolean connectives, regardless of what else it contains (e.g., an axiomatization of modal or probabilistic operators). We skip a formal definition, as propositional logics play a role only in our examples.

The agenda contains the formulas on which judgments are passed. Formally, it is any non-empty set $\Phi \subseteq \mathcal{L}$ that contains only contingent formulas and takes the form of a union of pairs $\{\varphi, \neg \varphi\}$, where $\varphi$ does not begin with $\neg$. From now on, when we write " $\neg \psi$ " with $\psi \in \Phi$, we mean the other element of the pair to which $\psi$ belongs. For any $S \subseteq \Phi$, we put $S^{ \pm}=\{\varphi, \neg \varphi: \varphi \in S\}$, and we call $S$ negation-closed if $S=S^{ \pm}$.

Elementary examples of agendas are $\Phi=\{a, b, a \rightarrow b\}^{ \pm}$and $\Phi=\{a, b, c, c \leftrightarrow(a \wedge b)\}^{ \pm}$ provide elementary examples of agendas in a propositional logic. The latter corresponds to the so-called doctrinal paradox, with $c$ representing the proposition that the defendant is liable, $a$ and $b$ the two legal conditions of liability, and $c \leftrightarrow(a \wedge b)$ the legal doctrine (see List and Pettit, 2002, for this classic example). A subagenda (of $\Phi$ ) is any non-empty negation-closed set $P \subseteq \Phi$. For instance, $P$ could consist of the literals contained in the agenda.

By assumption, any individual accepts some set $B \subseteq \Phi$ of formulas, his or her judgment set (JS). The theory of logical aggregation imposes universal constraints on acceptance, regardless of whether the accepted formulas represent, for instance, norms or facts. This and further internal distinctions might be reflected in the formalism, but we do not aim for this here.

A JS $B \subseteq \Phi$ is said to be complete w.r.t. $S \subseteq \Phi$ if for all $\varphi \in S$ it contains $\varphi$ or contains $\neg \varphi$, and to be deductively closed w.r.t. $S$ if for all $\varphi \in S$, it follows from $B \vdash \varphi$ that $\varphi \in B$. When these definitions hold for $S=\Phi$, we just call $B$ complete (resp. deductively closed). ${ }^{5}$

If a consistent JS is complete, then by (L2), (L3) and (L4) it is deductively closed, while the converse does not hold. Accordingly, we will focus on two graded notions of a JS $B \subseteq \Phi$, one in which $B$ is consistent and complete, and another in which $B$ is consistent and deductively closed. Let $D$ (resp. $D^{*}$ ) be the set of all JS of the first (resp. second) kind. Of course, $D \subsetneq D^{*}$. A JS in $D^{*}$ may be incomplete, even empty. Thus far, most authors have focused on the demanding case of completeness. Exceptions are Gärdenfors (2006), Dietrich and List (forthcoming) and Dokow and Holzman (2006).

Generally speaking, the theory means to investigate how individual judgments can be summarized into social judgments. In keeping with social choice theory, it uses mappings to represent aggregative rules, which it then characterizes in terms of

for epistemic applications are sometimes compact, and sometimes not, when they include a common belief operator (see Lismont and Mongin, 2003).

${ }^{5}$ Note that consistency and entailment remain defined in terms of the whole of $\mathcal{L}$, even when completeness and deductive closure are relativized to $\Phi$. A proof of $B \vdash \varphi$, where $B \cup\{\varphi\} \subseteq \Phi$, will involve formulas from $\mathcal{L} \backslash \Phi$ if only because $\Phi$ does not contain any theorem. 
axiomatic conditions put on these mappings. We follow this well-trodden path, but with a technical innovation: our set of individuals, to be denoted by $N$, may be of any cardinality $|N| \geq 2$, either finite or infinite. The current literature is limited to the former case. Accordingly, we define a social judgment function (SJF) to be a mapping

$$
F: D^{N} \rightarrow 2^{\Phi}
$$

with $D^{N} \rightarrow D$ and $D^{N} \rightarrow D^{*}$ as particular cases. The domain $D^{N}$ contains functions $N \rightarrow D$, which we typically denote $\left(A_{i}\right)_{i \in N}$ and refer to as profiles. An example - premiss-based majority voting - is given in a moment. In the finite case $N=$ $\{1, \ldots, n\}$, the domain $D^{N}$ reduces to the $n$-fold Cartesian product $D^{n}$, and profiles to $n$-tuples $\left(A_{1}, \ldots, A_{n}\right)$. Notice the unrestricted domain assumption that is implicit in the definition of a SJF.

\section{Premiss-based aggregation}

We represent the propositions singled out as premisses in terms of a fixed subagenda $P \subseteq \Phi$, and the premiss-based approach in terms of conditions bearing specifically on this set. The Latin letters $p, q, r, \ldots$ will be used for the formulas in $P$, also referred to as premisses. While the premiss-based procedure is typically considered restrictedly (see the introduction), we now define premiss-based majority voting for general $\Phi$ and $P$ (and finite $N$ ) as the following SJF $F_{P B M}: D^{N} \rightarrow 2^{\Phi}$. For any profile $\left(A_{i}\right)_{i \in N} \in D^{N}$, we first form the set $P_{\text {maj }}=\left\{p \in P:\left|\left\{i: p \in A_{i}\right\}\right|>|N| / 2\right\}$ of majority-accepted premisses, and then define $F_{P B M}\left(\left(A_{i}\right)_{i \in N}\right)$ as the entailment set $\left\{\varphi \in \Phi: P_{m a j} \vdash \varphi\right\}$ throughout the whole agenda. As one can check, $F_{P B M}$ generates social JS that are deductively closed, and consistent - so that $F_{P B M}: D^{N} \rightarrow D^{*}$ - if $P$ has no minimal inconsistent subset $Y$ with $|Y| \geq 3$.

A most salient property that $F_{P B M}$ possesses if $F: D^{N} \rightarrow D^{*}$ and $|N|$ is odd, is this

Independence on $P$. For all $p \in P$ and all $\left(A_{i}\right)_{i \in N},\left(A_{i}^{*}\right)_{i \in N} \in D^{N}$, if for all $i \in N, p \in A_{i} \Leftrightarrow p \in A_{i}^{*}$, then

$$
p \in F\left(\left(A_{i}\right)_{i \in N}\right) \Leftrightarrow p \in F\left(\left(A_{i}^{*}\right)_{i \in N}\right) .
$$

This condition will be applied throughout the paper. It requires that aggregation takes place formula by formula on $P$. It significantly weakens the Independence condition of the literature, to the effect that aggregation takes place formula by formula on the whole of $\Phi$.

We also impose the following classic requirement, this time on the whole of $\Phi$.

Unanimity-Preservation. For all $\varphi \in \Phi$ and all $\left(A_{i}\right)_{i \in N} \in D^{N}$, if for all $i \in N$, $\varphi \in A_{i}$, then $\varphi \in F\left(\left(A_{i}\right)_{i \in N}\right)$.

That unanimity should be preserved on $P$ seems to be a basic requirement on the premiss-based approach. That it is also preserved on $\Phi \backslash P$ sounds more like a hope, and a major objective of this section is to investigate whether the condition thus 
extended is compatible with our restricted independence condition. A mechanical effect of adding it is to exclude constant SJF, which trivially satisfy the latter.

Here are two classes of SJF that satisfy both Independence on $P$ and UnanimityPreservation. Define $F$ to be an oligarchy on $P$ if there is a non-empty $M \subseteq N$ - the oligarchs on $P$ - such that

$$
F\left(\left(A_{i}\right)_{i \in N}\right) \cap P=\cap_{i \in M}\left(A_{i} \cap P\right) \text { for all }\left(A_{i}\right)_{i \in N} \in D^{N},
$$

and to be a dictatorship on $P$ if $F$ is an oligarchy on $P$ with $M=\{i\}$ for some $i$ the dictator on $P$.

In the case $P=\Phi$, these definitions reduce to standard oligarchy and dictatorship notions; we then simply say "oligarchy" or "dictatorship", dropping "on $P$ ". ${ }^{6}$ Generally, if a rule, or a condition such as "Independence", is mentioned without adding

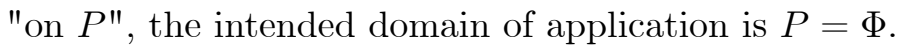

Whether any SJF for a finite society degenerates into dictatorship or oligarchy on $P$ when satisfying Independence on $P$ and Unanimity Preservation depends on how the premisses are logically connected, both with each other and with other formulas. As this section will show, three conditions - (a), (b) and (c) below - exactly describe the frontier between possibility and impossibility - in the sense of local dictatorship for a $D$-valued $F$, and local oligarchy for a $D^{*}$-valued $F$. The three conditions capture interconnections in terms of inconsistent sets and the following derivative notion: for any $\varphi, \psi \in \Phi, \varphi$ conditionally entails $\psi$ - formally, $\varphi \vdash^{*} \psi$ - if there is a set $Y \subseteq \Phi$ such that $Y \cup\{\varphi\} \vdash \psi$ and both $Y \cup\{\varphi\}$ and $Y \cup\{\neg \psi\}$ are consistent. The shorthand $Y_{\neg Z}$ denotes $(Y \backslash Z) \cup\{\neg \varphi: \varphi \in Z\}$, i.e., the set obtained from $Y$ by negating the formulas of one of its subsets $Z$.

\section{CONDITIONS ON PREMISSES:}

(a) There is a minimal inconsistent set $Y \subseteq \Phi$ such that $|Y \cap P| \geq 3$.

(b) There is a minimal inconsistent set $Y \subseteq \Phi$ such that $Y_{\neg Z}$ is consistent for some set $Z \subseteq Y \cap P$ of even cardinality.

(c) For all $p, q \in P$, there is a sequence $p_{1}, \ldots, p_{k} \in P(k \geq 2)$ such that $p=p_{1} \vdash^{*}$ $p_{2} \vdash^{*} \ldots \vdash^{*} p_{k}=q$.

These conditions relate to existing ones, but are unconventionally applied to only part of the agenda - the premisses. Detailed comparisons will be made in section 5. Importantly, (a), (b), (c) neither require, nor forbid the logical connections they describe to take place between the premisses alone. At one extreme, there could be none holding in terms of $P$ alone, except for the trivial ones between $p$ and $\neg p$. Then, all inconsistent $Y$ sets satisfying (a) and (b) contain non-premisses, and similarly with all the sets $Y_{1}, \ldots, Y_{k}$ supporting conditional entailments in (c). Such is the case under Mongin's (forthcoming) hypothesis that $P$ consists of logically independent p.v., but there are many other $P$ with the same pattern. To illustrate, take a propositional logic and $\Phi=\{a, a \rightarrow b, b\}^{ \pm}$, and $P=\{a, a \rightarrow b\}^{ \pm}$. Here, the members of $P$

\footnotetext{
${ }^{6}$ If $P=\Phi$, our oligarchy notion is that of Dietrich and List (forthcoming) and Dokow and Holzman (2006). Other writers use different notions of an oligarchy (Gärdenfors, 2006, Nehring and Puppe, forthcoming, Nehring, 2006).
} 
are not logically independent, but they become so when one replaces $a \rightarrow b$ by $a \hookrightarrow b$. Here and in later examples, we draw on properties of subjunctive (rather than material) implications, which hold at least in certain systems of conditional logic such as that used in Dietrich (forthcoming). The other extreme occurs when all relevant interconnections take place within $P$. This is automatically realized if $P=\Phi$, which is in effect the assumption of the literature, but again, there are many other instances.

None of the three conditions appears to be utterly strong. Condition (c) is compatible with a highly indirect linkage. Condition (a) is flexible, as the following case suggests: the logic is propositional and $P=\{a, b, c\}^{ \pm}$; then, (a) can be met by taking $\Phi$ to contain any of $a \vee b \vee c,(a \rightarrow(b \rightarrow c)),(a \leftrightarrow(b \leftrightarrow c))$, etc. As to (b), it is easy to satisfy it while not satisfying (a) and (c), e.g., if the logic is propositional and $\Phi=\{a, b, a \wedge b\}^{ \pm}$with $P=\{a, b\}^{ \pm}$. (Here, (b) holds with $Y=\{a, b, \neg(a \wedge b)\}$ and $Z=\{a, b\}$, while (a) fails as $P$ is too small, and (c) fails as, say, $\neg a$ does not conditionally entail any other premiss.)

To get a first flavour of why our conditions may narrow down the set of possible SJF, note that premiss-based majority voting $F_{P B M}$ violates Unanimity-Preservation if (a) holds (and $|N|$ is finite and not 2 or 4). Indeed, taking $Y$ as in (a), we can construct a profile $\left(A_{i}\right)_{i \in N} \in D^{N}$ with majorities for all premisses in $Y \cap P$ and a unanimity for each non-premiss in $Y \backslash P$.

We now state a representation theorem on premiss-based aggregation. It contains two results that differ in whether or not social judgment sets are required to be complete. Accordingly, the conclusions are graded in strength.

Theorem 1. Suppose that $N$ is finite with $|N| \geq 3$. If and only if (a)-(c) hold, every SJF $F: D^{N} \rightarrow D^{*}$ (resp. $D$ ) that is independent on $P$ and unanimitypreserving is an oligarchy (resp. a dictatorship) on $P$.

As the proof in the appendix makes clear, the sufficiency part of the theorem also holds for $|N|=2$. That part is easier to derive than the necessity part, which depends on elaborate counterexamples. Our proof is subdivided into nine lemmas stated in the main text. They make use of two further properties of SJF that need now introducing.

Systematicity on $P$. For all $p, p^{*} \in P$ and all $\left(A_{i}\right)_{i \in N},\left(A_{i}^{*}\right)_{i \in N} \in D^{N}$, if for all $i \in N, p \in A_{i} \Leftrightarrow p^{*} \in A_{i}^{*}$, then

$$
p \in F\left(\left(A_{i}\right)_{i \in N}\right) \Leftrightarrow p^{*} \in F\left(\left(A_{i}^{*}\right)_{i \in N}\right) .
$$

Monotonicity on $P$. For all $p \in P$ and all $\left(A_{i}\right)_{i \in N},\left(A_{i}^{*}\right)_{i \in N} \in D^{N}$, if for all $i \in N, p \in A_{i} \Rightarrow p \in A_{i}^{*}$, and for some $j \in N, p \notin A_{j}$ and $p \in A_{j}^{*}$, then

$$
p \in F\left(\left(A_{i}\right)_{i \in N}\right) \Rightarrow p \in F\left(\left(A_{i}^{*}\right)_{i \in N}\right) .
$$

By itself, the proof will bring to light three more results holding under the stated restriction on $N$. These are variants, not weakenings of the theorem because they involve weaker conditions on the premisses. 
(i) If and only if (a) and (b) hold, every SJF $F: D^{N} \rightarrow D^{*}$ (resp. $D$ ) that is systematic on $P$ and unanimity-preserving is an oligarchy (resp. a dictatorship) on $P$.

(ii) If and only if (a) holds, every SJF $F: D^{N} \rightarrow D$ (resp. $D^{*}$ ) that is systematic on $P$, monotonic on $P$, and unanimity-preserving, is an oligarchy (resp. a dictatorship) on $P$.

(iii) If and only if (a) and (c) hold, every SJF $F: D^{N} \rightarrow D^{*}$ (resp. D) that is independent on $P$, monotonic on $P$, and unanimity-preserving is an oligarchy (resp. a dictatorship) on $P$.

In a nutshell, Systematicity on $P$ makes it possible to dispense with condition (c), and Monotonicity on $P$ with condition (b). Does oligarchy (dictatorship) on $P$ imply oligarchy (dictatorship) simpliciter? This further question is reserved for section 5 .

In preparation of later sections, our lemmas allow $N$ to be infinite. As a tool, they use classic set-theoretical notions. For concreteness, we refer to subsets $C \subseteq N$ as coalitions. Now, a set of coalitions $\mathcal{D} \subseteq 2^{N}$ is superset-closed if for all coalitions $C, C^{*}, C \in \mathcal{D}$ and $C \subseteq C^{*} \subseteq N$ imply $C^{*} \in \mathcal{D}$; intersection-closed if for all coalitions $C, C^{*}, C, C^{*} \in \mathcal{D} \Rightarrow C \cap C^{*} \in \mathcal{D}$; coherent if for all coalitions $C, C \in \mathcal{D} \Rightarrow N \backslash C \notin \mathcal{D}$; complete if for all coalitions $C, C \notin \mathcal{D} \Rightarrow N \backslash C \in \mathcal{D}$; a filter if $\mathcal{D}$ is superset-closed and intersection-closed, with $\emptyset \notin \mathcal{D}$; an ultrafilter if $\mathcal{D}$ is a filter that is maximal for set-inclusion, or equivalently, complete. It follows that any filter is coherent, and non-empty only if $N \in \mathcal{D} .^{7}$

For any $\mathrm{SJF} F: D^{N} \rightarrow 2^{\Phi}$, let us say that $\mathcal{D} \subseteq 2^{N}$ generates $F$ on $p \in P$ if

$$
(*) \forall\left(A_{i}\right)_{i \in N} \in D^{N}, p \in F\left(\left(A_{i}\right)_{i \in N}\right) \Leftrightarrow\left\{i: p \in A_{i}\right\} \in \mathcal{D} .
$$

We then denote $\mathcal{D}$ by $\mathcal{C}_{p}^{F}$. This functional notation makes sense because there can be at most one such $\mathcal{D}$. If furthermore the same $\mathcal{D}$ generates $F$ on every $p \in P$, we say that $\mathcal{D}$ generates $F$ on $P$ and denote it by $\mathcal{C}^{F}$. For concreteness, we call the members of $\mathcal{C}^{F}(F$-)winning coalitions. When there is no ambiguity, we sometimes drop reference to $F$, writing $\mathcal{C}_{p}$ for $\mathcal{C}_{p}^{F}$ and $\mathcal{C}$ for $\mathcal{C}^{F}$.

To illustrate these notions, suppose that $F$ is an oligarchy on $P$ with a set of oligarchs $M \subseteq N$; then, $F$ is generated by $\mathcal{C}^{F}=\{C: M \subseteq C \subseteq N\}$. Less obvious examples will be introduced in section 4 .

Lemma 1. A SJF $F: D^{N} \rightarrow 2^{\Phi}$ is (i) independent on $P$ if and only if for every $p \in P$, there is $\mathcal{C}_{p}^{F} \subseteq 2^{N}$ generating $F$ on $p$, and (ii) systematic on $P$ if and only if there is $\mathcal{C}^{F} \subseteq 2^{N}$ generating $F$ on $P$.

An example of a SJF that is independent on $P$ but not systematic on $P$ is any constant rule, given by $F\left(\left(A_{i}\right)_{i \in N}\right)=\bar{A}$ for a fixed $\bar{A} \subseteq \Phi$. Here, $\mathcal{C}_{p}^{F}=2^{N}$ if $p \in \bar{A}$ and $\mathcal{C}_{p}^{F}=\emptyset$ if $p \notin \bar{A}$.

Lemma 2. Let a SJF $F: D^{N} \rightarrow D^{*}$ be independent on $P$. Then, $\mathcal{C}_{p}^{F}$ is coherent for all $p \in P$, and if moreover $F: D^{N} \rightarrow D$, then $\mathcal{C}_{p}^{F}$ is also complete for all $p \in P$. Also, $N \in \mathcal{C}_{p}^{F}$ and $\emptyset \notin \mathcal{C}_{p}^{F}$ if $F$ is unanimity-preserving.

\footnotetext{
${ }^{7}$ Filters are sometimes defined without requiring $\emptyset \notin \mathcal{D}$, in which case our filters are called proper filters. See Chang and Keisler (1973, p. 164).
} 
Lemma 3. Assume (b). Then, if a SJF $F: D^{N} \rightarrow D^{*}$ is systematic on $P$ and unanimity-preserving, $\mathcal{C}^{F}$ is superset-closed.

Lemma 4. Assume (a) and (b). Then, if a SJF $F: D^{N} \rightarrow D^{*}$ is systematic on $P$ and unanimity-preserving, $\mathcal{C}^{F}$ is intersection-closed.

Lemma 5. Assume (a) and (b). Then, if a SJF $F: D^{N} \rightarrow D^{*}$ (resp. D) is systematic on $P$ and unanimity-preserving, $\mathcal{C}^{F}$ is a filter (resp. ultrafilter), and if moreover $N$ is finite, $F$ is an oligarchy (resp. dictatorship) on $P$.

Lemma 5 implies the sufficiency part of Theorem 1 via a last lemma.

Lemma 6. If a SJF $F: D^{N} \rightarrow D^{*}$ is independent on $P$ and unanimity-preserving, then for all $p, q \in P, p \vdash^{*} q \Rightarrow \mathcal{C}_{p} \subseteq \mathcal{C}_{q}$; and if moreover (c) holds, $F$ is systematic on $P$.

As to the variants of Theorem 1, the sufficiency part of (i) was already proved at the stage of Lemma 5, while that of either (ii) or (iii) requires one to adapt the proofs of Lemmas 4 and 5 by replacing the assumption of (b) by the assumption that $F$ is monotonic on $P$. Inspection of these proofs show that they carry through.

The necessity part of Theorem 1 follows from the next three lemmas, because, as the reader can check, an oligarchy (or dictatorship) on $P$ is generated on $P$ by a filter $\mathcal{C}^{F}$.

Lemma 7. If $|N| \geq 3$ and (a) is violated, a SJF $F: D^{N} \rightarrow D$ exists that is systematic (hence independent) on $P$ and unanimity-preserving, and $\mathcal{C}^{F}$ is not a filter.

Lemma 8. If $|N| \geq 3$ and (b) is violated, a SJF $F: D^{N} \rightarrow D$ exists that is systematic (hence, independent) on $P$ and unanimity-preserving, and $\mathcal{C}^{F}$ is not a filter.

Lemma 9. If (c) is violated, a SJF $F: D^{N} \rightarrow D$ exists that is independent on $P$ and unanimity-preserving, and $\mathcal{C}_{p}^{F}$ is not the same for all $p \in P$.

\section{Filter rules and ultrafilter rules}

This section investigates the consequences for SJF of assuming that the number of individuals may be infinite. There are plausible reasons for recommending this step, which has been taken by social choice theorists already some time ago (in the wake of pathbreaking papers by Fishburn, 1970, and Kirman and Sonderman, 1972), but not yet by judgment aggregation theorists. If by "society" is meant a lasting community characterized by some non-transient features, such as manhood, the adherence to democracy, and the like, then social judgments should presumably be related to individual judgments that range across generations, and the infinite population assumption is prima facie relevant. Ultimately, our earlier conditions on $F$ should be 
reconsidered according to this intended interpretation, rather than just mechanically applied to a possibly infinite $N{ }^{8}$ This can be done step by step, and in this paper, we focus on the matter of (im)possibility under unchanged conditions on the SJF.

To include the infinite case, Theorem 1 needs reformulating in terms of broader classes of SJF. Let us define a SJF $F: D^{N} \rightarrow 2^{\Phi}$ to be a filter (resp. ultrafilter) rule on $P$ if it is generated on $P$ by a $\mathcal{C}^{F}$ that is a non-empty filter (resp. ultrafilter) on $N$. In view of what Lemmas 1-9 have achieved, we may reexpress Theorem 1 without the finiteness assumption in the following way.

Theorem 1'. Suppose that $|N| \geq 3$. If and only if (a)-(c) hold, every SJF $F: D^{N} \rightarrow D^{*}$ (resp. $D$ ) that is independent on $P$ and unanimity-preserving is a filter (resp. an ultrafilter) rule on $P$.

Theorem 1' has three variants without finiteness; they exactly parallel those of Theorem 1, with "filter rule" instead of "oligarchy" and "ultrafilter rule" of "dictatorship". One may now wonder whether for infinite $N$, Theorem 1' leaves room for non-degenerate possibilities under (a)-(c). Social choice theorists have long been aware that there exist non-empty filters over an infinite $N$ that are not principal - i.e., not of the form $\{C \subseteq N: M \subseteq C\}$ for any $M \subseteq N$. This follows from the fact that ultrafilters $\mathcal{C}$ over an infinite $N$ are divided into two non-empty classes, the principal ones and the so-called free ultrafilters, which satisfy $\cap_{C \in \mathcal{C}} C=\emptyset$. As an immediate consequence,

- for infinite $N$, not all filter (resp. ultrafilter) rules on $P$ are oligarchic (resp. dictatorial) on $P$.

But do these additional rules constitute genuine possibilities? We give a two-step answer. First, they satisfy the conditions that Theorem 1' places on SJF. To see that, consider filter (ultrafilter) rules that are defined on the whole of $\Phi$, and not just on $P$. These rules are trivially independent, even systematic, as well as unanimitypreserving (remember our convention of dropping "on $\Phi$ " when a condition or rule applies to this whole set). Non-trivially, thanks indeed to the compactness of the logic, they generate well-behaved social JS:

Proposition 1. Every filter (resp. ultrafilter) rule $F: D^{N} \rightarrow 2^{\Phi}$ is $D^{N} \rightarrow D^{*}$ $\left(\right.$ resp. $\left.D^{N} \rightarrow D\right)$.

It follows that the conclusion of Theorem 1' admits of a kind of converse. Start from any filter or ultrafilter $\mathcal{C}$ on $N$, including the non-principal ones, define the corresponding filter (ultrafilter) rule $F$, and finally modify it if necessary on $\Phi \backslash P$ to obtain a filter (an ultrafilter) rule on $P$ satisfying all the properties required from a SJF by Theorem 1'.

Second, we wish to exclude the fake possibilities in which an infinite, but intuitively unrepresentative group collectively dictates to the whole of $N$. In order to exclude these cases, we propose to use as a possibility criterion the existence of an anonymous rule, where anonymity will be defined in terms of suitable permutations.

\footnotetext{
${ }^{8}$ For the related social choice context, see, e.g., Lauwers's (1998) discussion of the Pareto condition.
} 
We will discuss anonymity notions that are analogous to ones put forward within preference aggregation. Since the distinction between premisses and other formulas is not of a primary importance for this discussion, we will assume that $P=\Phi$ for the rest of this section.

As a first try, we restrict attention to those permutations $\pi: N \rightarrow N$ which keep all except finitely many individuals unchanged, i.e., $\{i: \pi(i) \neq i\}$ is finite; we then call such a $\pi$ finite. Our normative requirement reads as follows.

Finite Anonymity. For all profiles $\left(A_{i}\right)_{i \in N} \in D^{N}$ and all finite permutations $\pi: N \rightarrow N, F\left(\left(A_{i}\right)_{i \in N}\right)=F\left(\left(A_{\pi(i)}\right)_{i \in N}\right)$.

Of course, Finite Anonymity excludes oligarchies, hence dictatorships, for whatever $N$, and becomes the plain anonymity condition (without restriction on $\pi$ ) if $N$ is finite. ${ }^{9}$ Far from being an unrealistic target, Finite Anonymity is easily achieved, as the following equivalence shows:

Proposition 2. If $N$ is infinite, the set of finitely anonymous ultrafilter rules is exactly the set of rules generated by free ultrafilters.

Propositions 1 and 2 together imply that

- if $N$ is infinite then (regardless of the logical connections within $\Phi$ ) there exist systematic and unanimity-preserving SJF $F: D^{N} \rightarrow D$ that are finitely anonymous.

But Finite Anonymity may seem too weak a condition on an infinite $N$ (for a related comment in social choice theory, see Lauwers and van Liedekerke, 1995). In response, one might require invariance under permutations that keep unchanged all but a set of individuals of cardinality at most $\kappa$, where $\kappa$ can be infinite but is smaller than $|N|$. If for instance $N=\mathbf{R}$ and $\kappa$ has countable cardinality, permutations can change all individuals with rational indexes, a clear improvement. Formally, we call $|\{i: \pi(i) \neq i\}|$ the cardinality of a permutation $\pi: N \rightarrow N$, and for any cardinal number $\kappa$, introduce the following requirement:

$\kappa$-Bounded Anonymity. For all profiles $\left(A_{i}\right)_{i \in N} \in D^{N}$ and all permutations $\pi: N \rightarrow N$ of cardinality up to $\kappa, F\left(\left(A_{i}\right)_{i \in N}\right)=F\left(\left(A_{\pi(i)}\right)_{i \in N}\right)$.

This stronger concept of anonymity also supports a possibility result, which, like the previous one, holds regardless of the logical connections within $\Phi$.

Proposition 3. If $N$ is infinite and $\kappa$ is any finite or infinite cardinal number smaller than $|N|$, there exist systematic and unanimity-preserving SJF $F: D^{N} \rightarrow D$ satisfying $\kappa$-Bounded Anonymity.

One can be dissatisfied even with this result, on the ground that the anonymity concept is still too weak. If $N=\mathbf{N}$, any cardinal $\kappa$ smaller than $|N|$ is finite, so

\footnotetext{
${ }^{9}$ Plain anonymity is used in List and Pettit's (2002) original theorem.
} 
that $\kappa$-Bounded Anonymity reduces to Finite Anonymity, ${ }^{10}$ hence cannot enforce the (intuitively desirable) equal treatment of $\{2 k: k \in \mathbf{N}\}$ and $\{2 k+1: k \in \mathbf{N}\}$. This critical observation suggests allowing permutations of any cardinality. But if all permutations are allowed, Theorem 1' implies a straightforward impossibility. E.g., any ultrafilter $\mathcal{F}$ on $\mathbf{N}$ must contain exactly one of the even and odd subgroups, which will lead any ultrafilter rule to violate anonymity. (Lauwers and van Liedekerke, 1995, have similar examples.)

In the hope of a response, one could endow $N$ with the structure of a measure space $(N, \mathcal{A}, \mu)$ and select those permutations $\pi$ which are measure-preserving, in the sense of being measurable and satisfying $\mu(\pi(C))=\mu(C)$ for all $C \in \mathcal{A}$. This move is also commendable in view of the intuition that cardinality-preservation cannot be all there is to anonymity. For instance, take $N=[0,1]$, equipped with the Borel$\sigma$-algebra $\mathcal{A}$ and the Lebesgue measure $\mu$; then, the measure-preserving requirement rids us of the pointless cardinality-preserving permutations $\pi: N \rightarrow N$ mapping $[0, .9]$ to $[0, .1]$. Hence the new requirement:

$(\mathcal{A}, \mu)$-Preserving Anonymity. For all profiles $\left(A_{i}\right)_{i \in N} \in D^{N}$ and all measurepreserving permutations $\pi: N \rightarrow N, F\left(\left(A_{i}\right)_{i \in N}\right)=F\left(\left(A_{\pi(i)}\right)_{i \in N}\right)$.

However, in the related Arrovian context, Kirman and Sonderman (1972, Proposition 5) have pointed out the following consequence of finite non-atomic measures $\mu$. By the convex-rangedness of property of such a measure, $N$ can be partitioned into finitely many cells $S_{1}, \ldots, S_{l} \in \mathcal{A}$ with $\mu\left(S_{1}\right)=\ldots=\mu\left(S_{l}\right) \leq \epsilon$, where $\epsilon$ is arbitrarily small. ${ }^{11}$ Since an ultrafilter must contain exactly one of $S_{k}$, an arbitrarily small coalition will be winning, which is undesirable. We connect this intuitive argument with our criterion of $(\mathcal{A}, \mu)$-Preserving Anonymity. For a violation to ensue, it is enough if some $\mu$-preserving permutation $\pi$ maps the winning $S_{k}$ to some nonwinning $S_{k^{\prime}}$. Take $N$ to be a bounded interval, $S_{1}, \ldots, S_{l}$ equally long subintervals, and $\mu$ the Lebesgue measure on the Borel sets of $N$.

By contrast, there are atomic measures that still allow for possibilities. A somewhat extreme case is that of a two-valued measure $\mu$ giving value 1 to all elements of a free ultrafilter $\mathcal{F}$ (this is a feasible example, as the reader will check). Here, the measurable winning coalitions are large in the chosen measure-theoretic sense, which distances the example from dictatorships and oligarchies.

An obvious objection to the measure-theoretic approach to anonymity is the potential arbitrariness involved in one's choosing $(\mathcal{A}, \mu)$. If $N=\mathbf{N}$, no finite measure $\mu$ on $\mathcal{A}=2^{N}$, except the zero-measure, gives all singletons $\{i\}$ the same measure; so that each choice of $\mu$ seems arbitrary if not objectionable. We may cautiously conclude that the possibility character of our results for infinite $N$ depends crucially on the notion of anonymity endorsed, and that much will depend on whether there exists a canonical choice of a measure space.

\footnotetext{
${ }^{10}$ The two conditions are rigorously equivalent for $\kappa \geq 2$, despite the restriction that $\kappa<|N|$. (Use the fact that every finite permutation is a composition of permutations of cardinality 2.)

${ }^{11}$ A measure $\mu$ on a measurable space $(N, \mathcal{A})$ is non-atomic if for all $S \in \mathcal{A}$, there is $S^{\prime} \in \mathcal{A}$ such that $0<\mu\left(S^{\prime}\right)<\mu(S)$. The property in the text follows from the more basic one that a non-atomic $\mu$ is convex-ranged.
} 


\section{Special sets of premisses and applications}

In this section, we first consider two special sets of premisses for which our theorems take a simpler form, and by the same token generalize several existing theorems to possibly infinite $N$ and $\Phi$. Second, we illustrate premiss-based aggregation with examples of $P$ that are fully general.

First, suppose that $P$ is the entire agenda $\Phi$. Then Independence on $P$ becomes standard Independence; (c) becomes total blockedness (introduced by Nehring and Puppe, 2002, later adopted by Dokow and Holzman, forthcoming); (b) becomes evennumber-negatability (Dietrich, 2007), which can be shown to be equivalent to nonaffineness (Dokow and Holzman) when $\Phi$ is finite, ${ }^{12}$ and finally, (a) can be dropped as it follows from total blockedness by an argument spelled out in Dokow and Holzman, after Nehring and Puppe.

Accordingly, the special case $P=\Phi$ of Theorem 1 can be reformulated thus.

Corollary 1. Let $|N| \geq 3$. If and only if the agenda is even-number-negatable and totally blocked, the independent and unanimity-preserving SJF $F: D^{N} \rightarrow D^{*}$ (resp. D) are exactly the filter (resp. ultrafilter) rules.

Since filter (resp. ultrafilter) rules are all that can be obtained, Corollary 1 and its three variants involving Systematicity and/or Monotonicity - their statements are left to the reader - generalize the corresponding dictatorship and oligarchy results of the literature. First of all, in the case of a $D$-valued SJF, Corollary 1 generalizes Dokow and Holzman's (forthcoming) important characterization of dictatorial agendas under Independence and Unanimity-Preservation, while the variants generalize dictatorship theorems proved under other assumptions. Second, in the case of a $D^{*}$-valued SJF, Corollary 1 generalizes oligarchy results by Dietrich and List (forthcoming), Dokow and Holzman (2006) and Gärdenfors (2006). Several of these results, including the first mentioned, are established for a finite set of formulas. We have gone beyond this assumption owing to an occasional use of compactness in the proofs. It is worth stressing that (L6) is needed only for the necessity part of our theorems.

By contrast, Corollary 1 has no direct bearing on those dictatorship results that do not include Unanimity-Preservation among their explicit conditions, e.g., those of Dietrich (2006, 2007), Dietrich and List (2005, 2007), and Pauly and van Hees (2006), van Hees (2007). Nor does Corollary 1 generalize oligarchy results by Dokow and Holzman (2006), Nehring (2006) and Nehring and Puppe (forthcoming), which are based on a different oligarchy notion. Vieille (2007) is similarly excluded because of his weak notion of systematicity.

Now we take up a question left unanswered in sections 3 and 4. Theorems 1 and 1 ' provide representations of the SJF that are local in the sense of being restricted to $P$. When do the local dictatorship or ultrafilter filter results translate into global ones? Here is the relevant condition:

(d) For all $\varphi \in \Phi$ and all $S \subseteq P$ that are consistent and complete w.r.t. $P$, either $S \vdash \varphi$ or $S \vdash \neg \varphi$.

\footnotetext{
${ }^{12} \mathrm{~A}$ proof of this equivalence is available on request.
} 
In view of the logical conditions on $\mathcal{L}$, it is equivalent to require that for all $B \in D$, $B=\{\varphi \in \Phi \mid B \cap P \vdash \varphi\}$, i.e., that any JS $B$ in $D$ can be recovered from its premisses by entailment. The distinction between whether or not (d) holds is similar to Nehring and Puppe's (2007, forthcoming,) distinction between truth-functional and non-truthfunctional agendas; but these authors apply an unrestricted independence condition, so that no clear connection exists to their results.

Obviously, under (d) a dictator on $P$ is a dictator. Perhaps not so obviously, under (d) every ultrafilter rule on $P$ is also an ultrafilter rule, as shown below. This implies the sufficiency part of the following theorem.

Theorem 2. Suppose $|N| \geq 3$. If and only if (a)-(d) hold, the SJF $F: D^{N} \rightarrow D$ that are independent on $P$ and unanimity-preserving are exactly the ultrafilter rules.

Of course, "ultrafilter rules" can be replaced by "dictatorships" if $N$ is finite. To derive the sufficiency and necessity parts of Theorem 2 from the corresponding parts of Theorem 1', we draw on two more lemmas.

Lemma 10. Assume (d) holds. If a SJF $F: D^{N} \rightarrow D$ is an ultrafilter rule on $P$, it is an ultrafilter rule.

Lemma 11. If (d) is violated, a SJF $F: D^{N} \rightarrow D$ exists that is systematic (hence independent) on $P$ and unanimity-preserving, but not a filter rule.

As before, we leave it for the reader to devise the respective variants after those of Theorem 1.

A fully generating set of premisses $P$, in the sense required by (d), should be seen as an exception rather than the rule, which severely constrains the relevance of Theorem 2 and its variants. With the doctrinal paradox agenda $\Phi=\{a, b, c \leftrightarrow(a \wedge b), c\}^{ \pm}$, (d) fails if $P=\{a, b\}^{ \pm}$, but not if $P=\{a, b, c \leftrightarrow(a \wedge b)\}$. Now, take a propositional conditional logic, replacing the material biconditional $\leftrightarrow$ by a subjunctive one $\hookleftarrow \hookrightarrow$. Then, even the enlarged $P$ does not fully generate $\Phi$, because, say, of the JS $\{\neg a, b, c \hookleftarrow \hookrightarrow(a \wedge b), \neg c\}$.

For the sake of generality, we may consider a partly generating $P$, as defined by

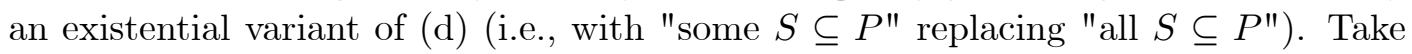
$N$ to be finite. If (a)-(c) and this variant hold, then any $F$ meeting the conditions will not need to be a dictatorship on the full domain $D^{N}$, but must be one on the non-empty subdomain consisting of all profiles $\left(A_{i}\right)_{i \in N}$ the images of which $F\left(A_{i}\right)_{i \in N}$ happen to be entailed by their premisses. For instance, if $P=\{a, c, c \leftrightarrow(a \wedge b)\}^{ \pm}$, a case where (d) fails but its existential variant holds, some individual $j$ dictates on $\Phi$ for profiles $\left(A_{i}\right)_{i \in N} \in D^{N}$ in which

$$
A_{j}=\{a, c, c \leftrightarrow(a \wedge b), \ldots\},
$$

but not for profiles $\left(A_{i}\right)_{i \in N} \in D^{N}$ in which

$$
A_{j}=\{\neg a, \neg c, c \leftrightarrow(a \wedge b), \ldots\} .
$$


While the above-discussed literature takes $P=\Phi$, Mongin (forthcoming) takes $P$ to be a very restricted subagenda of $\Phi$, i.e., the set of literals of an agenda $\Phi \subseteq$ $\mathcal{L}$, where $\mathcal{L}$ is the language of classical propositional logic. Then, Independence on $P$ becomes Independence of Irrelevant Propositional Alternatives, which Mongin shows to imply dictatorship in the presence of Unanimity Preservation. This theorem can also be recovered from Theorem 2, because its agenda conditions are (a), local asymmetry, which strengthens (b), (c), and closure under propositional variables, which implies $(\mathrm{d})$ given the choice of the logical language. The latter closure condition, which is also used by Pauly and van Hees (2006), requires $P$ to contain every p.v. $a \in \mathcal{L}$ occurring in some $\varphi \in \Phi$. It becomes equivalent to (d) if "occurring in" is replaced by "occurring essentially in" (so as to exclude, e.g., $a$ in $b \wedge(a \vee \neg a)$ ).

We conclude by discussing a type of set of premisses that seems to occur frequently in effective applications; it will also illustrate that our theorems are fruitful on the possibility side. For a propositional conditional logic, take $\Phi$ and $P$ such that $\Phi \backslash P$ contains only literals, typically representing propositions that are policy- or actionoriented (such as "the defendant is liable", which entails convicting the defendant). Suppose further that $P$ consists of the following:

1. Literals, typically stating basic facts or norms, or reasons for or against certain policies or actions.

2. Subjunctive implications $p \hookrightarrow q$, or negations thereof, with $p$ a type- 1 premiss or any Boolean expression built from type-1 premisses, and $q$ a member of $\Phi \backslash P$ or any Boolean expression built from members of $\Phi \backslash P$.

So type-2 premisses state that certain (combinations of) type- 1 premisses $p$ have implications $q$ on the non-premisses. Note that whether and how judgments on nonpremisses are logically constrained by judgments on premisses depends on what kind of type-2 premisses are hypothesized in the given JS. By conditional logic, if the selected premisses are of the negated type 2, nothing can be inferred on $\Phi \backslash P$. This already implies that (d) does not hold, hence from Theorem 2 that there exist nondictatorial SJF that are independent on $P$ and unanimity-preserving. Condition (c) does not hold either, because in (many systems of) conditional logic, a negated type- 2 premiss cannot conditionally entail any premiss other than a negated type- 2 premiss. Thus, from Theorem 1, there exist SJF that are well-behaved even on $P$.

By contrast, conditions (a) and (b) can easily be met with the present type of set of premisses, so that the impossibilities of the first two variants of Theorem 1 do apply. Suppose for instance that $p \hookrightarrow q \in P$ and $q \in \Phi$. Then there is a consistent set $A$ of type- 1 premisses that is minimal subject to entailing $p$ (e.g. $A=\{a, \neg b\}$ if $p$ is $a \wedge \neg b)$. Then, $Y=A \cup\{p \hookrightarrow q, \neg q\}(\subseteq \Phi)$ is minimal inconsistent. Condition (b) holds because there is $Z \subseteq Y \cap P$ consisting of $p \hookrightarrow q$ and any one member of $A$. If $|A| \geq 2$, condition (a) holds too because $|Y \cap P|=|A \cup\{p \hookrightarrow q\}| \geq 3$. By the first two variants of Theorem 1, various plausible rules degenerate. In particular, premiss-based majority voting cannot be applied here. However, in other instances of the same type, including many with logically independent premisses, (a) or (b) fails, and premiss-based majority voting is well-behaved. 


\section{Proofs}

Notation. When a profile $\left(A_{i}\right)_{i \in N}$ is given, we often write $A$ instead of $F\left(\left(A_{i}\right)_{i \in N}\right)$, and for $p \in P, N_{p}$ instead of $\left\{i \in N: p \in A_{i}\right\}$. For $Z \subseteq \Phi$, we denote $\{\neg p: p \in Z\}$ by $\neg Z$.

Proof of Lemma 1. Associate with the SJF $F: D^{N} \rightarrow 2^{\Phi}$ and possibly with $p \in P$ the following sets of coalitions:

$$
\begin{aligned}
& \mathcal{D}_{p}=\left\{C \subseteq N: \exists\left(A_{i}\right)_{i \in N} \in D^{N},\left\{i: p \in A_{i}\right\}=C \& p \in F\left(\left(A_{i}\right)_{i \in N}\right)\right\}, \\
& \overline{\mathcal{D}}_{p}=\left\{C \subseteq N: \forall\left(A_{i}\right)_{i \in N} \in D^{N},\left\{i: p \in A_{i}\right\}=C \Rightarrow p \in F\left(\left(A_{i}\right)_{i \in N}\right)\right\},
\end{aligned}
$$

and

$$
\mathcal{D}=\left\{C \subseteq N: \forall p \in P, \forall\left(A_{i}\right)_{i \in N} \in D^{N},\left\{i: p \in A_{i}\right\}=C \Rightarrow p \in F\left(\left(A_{i}\right)_{i \in N}\right)\right\} .
$$

Clearly, $\mathcal{D} \subseteq \overline{\mathcal{D}}_{p} \subseteq \mathcal{D}_{p}$. It is easy to see that $F$ is independent on $P$ if and only if $\mathcal{D}_{p}=\overline{\mathcal{D}}_{p}$. Now, if this equality holds, $\overline{\mathcal{D}}_{p}$ satisfies condition $(*)$ of the text. Hence, if $F$ is independent on $P$, there exists $\mathcal{C}_{p}$ generating $F$ on $p$. The converse implication is trivial. By the same token, $F$ is systematic on $P$ if and only if $\mathcal{D}=\mathcal{D}_{p}$ for all $p \in P$. But if these equalities hold, condition $(*)$ holds for the same set regardless of $p$. Hence, if $F$ is systematic on $P$, there exists $\mathcal{C}^{F}$ generating $F$ on $P$. The converse is trivial.

Proof of Lemma 2. Left to the reader.

Proof of Lemma 3. Let (b) hold. We first derive a consequence of (b), and then proceed to the proof itself.

Claim. There exist an inconsistent set $W \subseteq \Phi$ and disjoint sets $V,\{p\} \subseteq W \cap P$ such that $W_{\neg V}, W_{\neg\{p\}}$ and $W_{\neg(V \cup\{p\})}$ are consistent.

Let $Y$ be as in (b), and choose a $Z \subseteq Y \cap P$ of smallest even size subject to $Y_{\neg Z}$ being consistent. There are two cases.

Case 1: $Y_{\neg Z^{\prime}}$ is consistent for some $Z^{\prime} \subseteq Z$ of size $|Z|-1$. Then, the claim holds for $W=Y, V=Z^{\prime}$ and $\{p\}=Z \backslash Z^{\prime}$ (where $W_{\neg\{p\}}$ is consistent because $Y$ is minimal inconsistent).

Case 2: $Y_{\neg Z^{\prime}}$ is inconsistent for all $Z^{\prime} \subseteq Z$ of size $|Z|-1$. Then, $|Z| \geq 4$, as $|Z|$ is even, and neither zero (since $Y$ is inconsistent) nor 2 (because this and the minimal inconsistency of $Y$ would lead to a consistent $Y_{\neg Z^{\prime}}$ ). In particular, $Y$ contains no pair $r, \neg r$ (something to be implicitly used later). Consider distinct $p, q \in Z$, and put

$$
V=\neg(Z \backslash\{p, q\}), W=(Y \backslash Z) \cup\{p\} \cup V .
$$

The claim holds of $W, V$ and $\{p\}$ so defined for the following reasons:

- $\{p\}$ and $V$ are disjoint subsets of $W \cap P$;

- $W$ is inconsistent as $W \cup\{q\}=Y_{\neg(Z \backslash\{p, q\})}$ is inconsistent by the minimality property of $Z$, and $W \cup\{\neg q\}=Y_{\neg(Z \backslash\{p\})}$ is inconsistent by the case-2 assumption;

- $W_{\neg\{p\}}$ is consistent as a subset of the consistent $Y_{\neg Z}$; 
- $W_{\neg V}=Y \backslash\{q\}$ and $W_{\neg(\{p\} \cup V)}=Y_{\neg\{p\}} \backslash\{q\}$ are consistent because $Y$ is minimal inconsistent (for the latter, consider the superset $Y_{\neg\{p\}}$ ).

Main proof. Take $F$ as specified, with associated $\mathcal{C}$. We assume that $C \in \mathcal{C}$ and $C \subseteq C^{*} \subseteq N$ and show that $C^{*} \in \mathcal{C}$. Now, the restatement of $\mathcal{C}$ in the proof of Lemma 1 means that it is enough for the proof to select a particular $\left(A_{i}\right)_{i \in N} \in D^{N}$ and a particular $r \in P$ s.t.

$$
C=N_{r} \text { and } r \in A \text {. }
$$

Relying on the claim, we extend the consistent sets $W_{\neg\{p\}}, W_{\neg V}$ and $W_{\neg(V \cup\{p\})}$ to JS in $D$, resp. $A_{W_{\neg\{p\}}}, A_{W_{\neg V}}$ and $A_{W_{\neg(V \cup\{p\})}}$, and consider the profile $\left(A_{i}\right)_{i \in N} \in D^{N}$ defined by

$$
A_{i}= \begin{cases}A_{W_{\neg\{p\}}} & \text { if } i \in C \\ A_{W_{\neg(V \cup\{p\})}} & \text { if } i \in C^{*} \backslash C \\ A_{W_{\neg V}} & \text { if } i \in N \backslash C^{*} .\end{cases}
$$

Now, $A$ contains all $q \in V$, since then $N_{q}=C \in \mathcal{C}$, and all $q \in W \backslash(V \cup\{p\})$, since then $N_{q}=N \in \mathcal{C}$ by Lemma 2 . So $W \backslash\{p\} \subseteq A$. The inconsistency of $W$ ensures that $W \backslash\{p\} \vdash \neg p$, whence $\neg p \in A$ by the assumption that $A \in D^{*}$. So $\left\{i: \neg p \in A_{i}\right\} \in \mathcal{C}$, which implies that $C^{*} \in \mathcal{C}$ as desired.

Proof of Lemma 4. Assume (a) and (b). Take $F$ as specified, the associated $\mathcal{C}$, and any $C, C^{*} \in \mathcal{C}$. Take $Y \subseteq \Phi$ as in (a). There are at least three pairwise distinct formulas $p, q, r \in Y \cap P$, and the sets $Y_{\neg\{p\}}, Y_{\neg\{q\}}$ and $Y_{\neg\{r\}}$ are consistent by the minimal inconsistency of $Y$. Hence, there is $\left(A_{i}\right)_{i \in N} \in D^{N}$ as follows:

- for all $i \in C \cap C^{*}, A_{i}$ extends $Y_{\neg\{p\}}$,

- for all $i \in C^{*} \backslash C, A_{i}$ extends $Y_{\neg\{r\}}$,

- for all $i \in N \backslash C^{*}, A_{i}$ extends $Y_{\neg\{q\}}$.

Unanimity Preservation ensures that $Y \backslash\{p, q, r\} \subseteq A$. Further, $q \in A$ because $N_{q}=\left(C \cap C^{*}\right) \cup\left(C^{*} \backslash C\right)=C^{*} \in \mathcal{C}$, and $r \in A$ because $N_{r}=\left(C \cap C^{*}\right) \cup\left(N \backslash C^{*}\right) \supseteq$ $C \in \mathcal{C}$ and $\mathcal{C}$ is superset-closed by Lemma 3. Thus, $Y \backslash\{p\} \subseteq A$, and $\neg p \in A$ since $Y$ is inconsistent and $A \in D^{*}$. This is sufficient for the conclusion that $C \cap C^{*} \in \mathcal{C}$, as was to be proved.

Proof of Lemma 5. Assume (a) and (b). Take $F$ as specified and the associated $\mathcal{C}$. From Lemma 2, $\mathcal{C}$ does not contain $\emptyset$, and from Lemmas 3 and $4, \mathcal{C}$ is superset- and intersection-closed. Hence $\mathcal{C}$ is a filter. If moreover $F: D^{N} \rightarrow D$, Lemma 2 implies the stronger conclusion that $\mathcal{C}$ is an ultrafilter. As is well-known, if $N$ is finite, every filter is the set of supersets of some $M \subseteq N$, and every ultrafilter the set of supersets of $\{i\}$ for some $i \in N$; so that $F$ is either an oligarchy or a dictatorship, respectively.

Proof of Lemma 6. For $F$ as specified, consider $p, q \in P$ and the associated $\mathcal{C}_{p}, \mathcal{C}_{q}$. Take $p \vdash^{*} q$, and let $C \in \mathcal{C}_{p}$. By definition of $\vdash^{*}$, there is $Y \subseteq \Phi$ s.t. $Y \cup\{p\}$ and $Y \cup\{\neg q\}$ are consistent, and $Y \cup\{p, \neg q\}$ is inconsistent. As the last claim implies, $Y \cup\{p, q\}$ and $Y \cup\{\neg p, \neg q\}$ are consistent, and there exists $\left(A_{i}\right)_{i \in N} \in D^{N}$ as follows:

- for all $i \in C, A_{i}$ extends $Y \cup\{p, q\}$, 
- for all $i \notin C, A_{i}$ extends $Y \cup\{\neg p, \neg q\}$.

With this profile, $Y \subseteq A$ by Unanimity Preservation, and $p \in A$ because $\{i: p \in$ $\left.A_{i}\right\} \in \mathcal{C}_{p}$. So $q \in A$ since $A \in D^{*}$. By $N_{q}=C$ and $q \in A$, we have that $C \in \mathcal{C}_{q}$, as was to be proved.

Suppose now that (c) holds. Then, for all $p, q \in P$, the sequence of conditional entailments $p \vdash^{*} p_{2}, \ldots, p_{k-1} \vdash^{*} q$ made available by this condition leads to a corresponding sequence of inclusions $\mathcal{C}_{p} \subseteq \mathcal{C}_{p_{2}}, \ldots, \mathcal{C}_{p_{k-1}} \subseteq \mathcal{C}_{q}$, and then to $\mathcal{C}_{p}=\mathcal{C}_{q}$, so that by Lemma $1, F$ is systematic on $P$.

Proof of Lemma \%. Let $|N| \geq 3$, and let (a) be violated. Then there is an odd-sized coalition $M \subseteq N$ with $|M| \neq 1$. For any $\left(A_{i}\right)_{i \in N} \in D^{N}$, we define the set

$$
B=\left(\cap_{i \in N} A_{i}\right) \cup\left\{p \in P:\left|\left\{i \in M: p \in A_{i}\right\}\right|>|M| / 2\right\} .
$$

(In words, $B$ collects all formulas unanimously accepted and all premisses accepted by a majority within $M$.) We will show that $B$ is consistent. If not, by (L6), $B$ has a finite minimal inconsistent subset $Y \subseteq B$. As (a) does not hold, $|Y \cap P| \leq 2$, hence $|Y \cap P|=2$ since $\Phi$ contains no contradictions. Say $Y \cap P=\{p, q\}$. Within $M$, a majority accepts $p$, and a majority accepts $q$. As two majorities must overlap, there is an $j \in M$ s.t. $\{p, q\} \subseteq A_{j}$. So $Y \cap P \subseteq A_{j}$. Hence, as also $Y \backslash P \subseteq A_{j}, Y \subseteq A_{j}$. So $A_{i}$ is inconsistent, a contradiction.

Having just shown that $B$ is consistent, $B$ can be extended to a set in $D$; let $F\left(\left(A_{i}\right)_{i \in N}\right)$ be one such extension. Note that, as $B$ is already complete w.r.t. $P$,

$$
F\left(\left(A_{i}\right)_{i \in N}\right) \cap P=B \cap P=\left\{p \in P:\left|\left\{i \in M: p \in A_{i}\right\}\right|>|M| / 2\right\} .
$$

So we have defined a SJF $F: D^{N} \rightarrow D$ that is generated on $P$ by $\mathcal{C}=\{C \subseteq M:|C|>$ $|M| / 2\}$. Lemma 1 implies that $F$ is systematic. Also, $F$ is unanimity-preserving by construction. And $\mathcal{C}$ is not a filter, because $\mathcal{C}$ is not intersection-closed (take, e.g., two majorities of $\frac{|M|+1}{2}$ individuals that intersect on a singleton).

Proof of Lemma 8. Let $|N| \geq 3$; so $N$ contains three distinct individuals, to be labelled 1,2,3. Let (b) be violated. For any $\left(A_{i}\right)_{i \in N} \in D^{N}$, we define $B=B_{1} \cup B_{2}$, where

$$
B_{1}=A_{1} \cap A_{2} \cap A_{3}
$$

and

$$
B_{2}=\left\{p \in P: p \text { is in exactly one of } A_{1}, A_{2}, A_{3}\right\} .
$$

We prove that $B$ is consistent. Suppose not, then by (L6) there is a finite minimal inconsistent subset $Y \subseteq B$. Define $Y^{*}=Y \cap P$ and $A_{i}^{*}=A_{i} \cap P$ for all $i \in N$. We have $Y^{*} \neq \emptyset$, as otherwise $Y \subseteq B_{1} \subseteq A_{1}$, an impossibility since $Y$ is inconsistent and $A_{1}$ is consistent. Now, $Y^{*}$ can be expressed as the pairwise disjoint union of the following sets:

$$
\begin{aligned}
& Z_{0}=Y^{*} \cap A_{1}^{*} \cap A_{2}^{*} \cap A_{3}^{*}, Z_{1}=Y^{*} \cap\left[A_{1}^{*} \backslash\left(A_{2}^{*} \cup A_{3}^{*}\right)\right], \\
& Z_{2}=Y^{*} \cap\left[A_{2}^{*} \backslash\left(A_{1}^{*} \cup A_{3}^{*}\right)\right], Z_{3}=Y^{*} \cap\left[A_{3}^{*} \backslash\left(A_{1}^{*} \cup A_{2}^{*}\right)\right] .
\end{aligned}
$$


There must exist two sets among $Z_{1}, Z_{2}, Z_{3}$, say w.l.g. $Z_{1}, Z_{2}$, such that $\left|Z_{1} \cup Z_{2}\right|$ is even and $Z_{1} \cup Z_{2} \neq \emptyset$. (The first claim is simply combinatorial, and the second one follows by contradiction from the consistency of $A_{3}$, since $Z_{1} \cup Z_{2}=\emptyset$ leads to $Y^{*} \subseteq A_{3}$, hence to $Y \subseteq A_{3}$.) Put $Z=Z_{1} \cup Z_{2}$. Since (b) does not hold, we will have derived a contradiction if we show that

$$
Y_{\neg Z}=(Y \backslash Z) \cup \neg Z
$$

is consistent. Now, $Y_{\neg Z}$ can be obtained as the union

$$
Y=\left(Y \backslash Y^{*}\right) \cup\left(Y^{*} \backslash Z\right) \cup \neg Z,
$$

where (i) $Y \backslash Y^{*} \subseteq B_{1} \subseteq A_{3}$, (ii) $Y^{*} \backslash Z=Y^{*} \cap A_{3}^{*} \subseteq A_{3}^{*}$, and (iii) $\neg Z=\left\{\neg p: p \in Y^{*}, p \notin A_{3}^{*}\right\} \subseteq$ $\left\{\neg p: p \in P, p \notin A_{3}^{*}\right\}=A_{3}^{*}$. The last equality holds as $A_{3}^{*}=A_{3} \cap P$ contains exactly one member of each pair $p, \neg p \in P$. Putting (i), (ii) and (iii) together, we see that $Y_{\neg Z} \subseteq A_{3}$, hence that $Y_{\neg Z}$ is consistent, as we aimed at proving.

Having shown $B$ to be consistent, we can extend $B$ to a set in $D$; this set is our $F\left(\left(A_{i}\right)_{i \in N}\right)$. Note that, as $B$ is already complete w.r.t. $P$,

$$
F\left(\left(A_{i}\right)_{i \in N}\right) \cap P=B \cap P=\left\{p \in P:\left|N_{p}\right| \text { is odd }\right\} .
$$

So the just-defined SJF $F: D^{N} \mapsto D$ is generated on $P$ by

$$
\mathcal{C}^{F}=\{C \subseteq N:|C \cap\{1,2,3\}| \text { is odd }\} .
$$

Hence, $F$ is systematic on $P$ from Lemma 1 ; it is also unanimity-preserving since $\cap_{i \in N} A_{i} \subseteq B_{1} \subseteq F\left(\left(A_{i}\right)_{i \in N}\right)$. But $\mathcal{C}^{F}$ is not a filter, as it is not superset-closed.

Proof of Lemma 9. Let (c) be violated. As $|N| \geq 2, N$ contains distinct individuals, to be labelled 1 and 2. For $p, q \in P$, define $p R q$ if there is a sequence of conditional entailments from $p$ to $q$ as in the statement of (c). As (c) does not hold, there are $\bar{p}, \bar{q} \in P$ such that not $\bar{p} R \bar{q}$, and $P$ can be partitioned into two non-empty sets

$$
S_{1}=\{p \in P: \bar{p} R p\} \text { and } S_{2}=\{p \in P: \operatorname{not} \bar{p} R p\}
$$

Note that

$$
p \not \nvdash^{*} q \text { for all } p \in S_{1} \text { and all } q \in S_{2} .
$$

We can further partition $S_{1}$ into the sets

$$
S_{11}=\left\{p \in S_{1}: \neg p \in S_{1}\right\} \text { and } S_{12}=\left\{p \in S_{1}: \neg p \in S_{2}\right\},
$$

and similarly, $S_{2}$ into the sets

$$
S_{21}=\left\{p \in S_{2}: \neg p \in S_{1}\right\} \text { and } S_{22}=\left\{p \in S_{2}: \neg p \in S_{2}\right\} .
$$

Now, consider any $\left(A_{i}\right)_{i \in N} \in D^{N}$. We first define $B \subseteq P$ as follows: for all $p \in P$,

$$
p \in B \Leftrightarrow \begin{cases}p \in A_{1} & \text { if } p \in S_{11} \\ p \in A_{2} & \text { if } p \in S_{22} \\ p \in A_{1} \cup A_{2} & \text { if } p \in S_{12} \\ p \in A_{1} \cap A_{2} & \text { if } p \in S_{21} .\end{cases}
$$


We set out to prove that $B \cup\left(A_{1} \cap A_{2}\right)$ is a consistent set. Suppose not; then by (L6), there is a minimal inconsistent subset $Y \subseteq B \cup\left(A_{1} \cap A_{2}\right)$. Hence,

$$
p \vdash^{*} \neg q \text { for all distinct } p, q \in Y \text {. }
$$

We will prove six claims relative to $Y^{*}=Y \cap B$, leading eventually to a contradiction.

(i) $Y^{*} \nsubseteq S_{11} \cup S_{21}$. If not, the definition of $B$ implies that $Y^{*} \subseteq A_{1}$, and $Y \subseteq A_{1}$, a consistent set.

(ii) $Y^{*} \nsubseteq S_{22} \cup S_{21}$ by a similar argument.

(iii) $Y^{*} \cap S_{12} \neq \emptyset$. If not, $Y^{*} \subseteq S_{11} \cup S_{22} \cup S_{21}$, and by (i) and (ii), there are $p, q \in Y^{*}$ with $p \in S_{11}$ and $q \in S_{22}$, hence also $\neg q \in S_{22}$. By (2), $p \vdash^{*} \neg q$, contradicting $(1)$.

(iv) $Y^{*} \cap S_{12}=\{r\}$. If there were $r, s \in Y^{*} \cap S_{12}, r \neq s$, (2) would imply that $s \vdash^{*} \neg r$, in contradiction with (1).

(v) $Y^{*} \cap S_{11}=\emptyset$. If not, by (2) $p \vdash^{*} \neg r$, contradicting (1).

(vi) $Y^{*} \cap S_{22}=\emptyset$ by a similar argument.

From (iv), (v) and (vi), $Y^{*} \subseteq\{r\} \cup S_{21} \subseteq\{r\} \cup\left(A_{1} \cap A_{2}\right)$, where the second inclusion follows from the definition of $B$. Since $Y \subseteq Y^{*} \cup\left(A_{1} \cap A_{2}\right)$, it also holds that $Y \subseteq\{r\} \cup\left(A_{1} \cap A_{2}\right)$. The definition of $B$ implies that $r \in A_{1}$ or $r \in A_{2}$, whence either $Y \subseteq A_{1}$ or $Y \subseteq A_{2}$, a contradiction with the consistency of $A_{1}$ and $A_{2}$.

For all $\left(A_{i}\right)_{i \in N} \in D^{N}$, one can extend the consistent set $B \cup\left(A_{1} \cap A_{2}\right)$ to one in $D$, so as to define a SJF $F: D^{N} \rightarrow D$. As $B$ was already complete w.r.t. $P$, we have $F\left(\left(A_{i}\right)_{i \in N}\right) \cap P=B \cap P$ for all $\left(A_{i}\right)_{i \in N} \in D^{N}$. It follows that, for every $p \in P, F$ is generated on $p$ by some $\mathcal{C}_{p}$, hence by Lemma 1 that $F$ is independent on $P$. $F$ is unanimity-preserving since $\cap_{i \in N} A_{i} \subseteq A_{1} \cap A_{2} \subseteq A$. Finally, $\mathcal{C}_{p}$ is not the same for all $p \in P$, because $S_{1}$ and $S_{2}$ are each non-empty, and if $p \in S_{1}$ then $\mathcal{C}_{p}$ is $\{C \subseteq N: 1 \in C\}$ or $2^{N} \backslash\{\emptyset\}$, whereas if $p \in S_{2}$ then $\mathcal{C}_{p}$ is $\{C \subseteq N: 2 \in C\}$ or $\{N\}$.

Proof of Lemma 10. Suppose (d) holds and $F: D^{N} \rightarrow D$ is an ultrafilter rule on $P$, with ultrafilter $\mathcal{C}$. Let $G: D^{N} \rightarrow 2^{\Phi}$ be the ultrafilter rule generated by $\mathcal{C}$. By Lemma $2, G: D^{N} \rightarrow D$. To show that $F=G$, we consider any $\left(A_{i}\right)_{i \in N} \in D^{N}$ and show that $F\left(\left(A_{i}\right)_{i \in N}\right)=G\left(\left(A_{i}\right)_{i \in N}\right)$. As $F\left(\left(A_{i}\right)_{i \in N}\right)$ and $G\left(\left(A_{i}\right)_{i \in N}\right)$ and each in $D$, it suffices to show that $F\left(\left(A_{i}\right)_{i \in N}\right) \subseteq G\left(\left(A_{i}\right)_{i \in N}\right)$. Consider any $\phi \in F\left(\left(A_{i}\right)_{i \in N}\right)$. Let $S:=F\left(\left(A_{i}\right)_{i \in N}\right) \cap P$. By (d), either $S \vdash \phi$ or $S \vdash \neg \phi$. It cannot be that $S \vdash \neg \phi$, since otherwise $F\left(\left(A_{i}\right)_{i \in N}\right)$ would contain $\neg \phi$ (by deductive closure), hence be inconsistent. So $S \vdash \phi$. By definition of $G, G\left(\left(A_{i}\right)_{i \in N}\right) \cap P=F\left(\left(A_{i}\right)_{i \in N}\right) \cap P$, whence $G\left(\left(A_{i}\right)_{i \in N}\right) \cap P=S$. So also $G\left(\left(A_{i}\right)_{i \in N}\right)$ entails $\phi$. Hence, as $G\left(\left(A_{i}\right)_{i \in N}\right)$ is deductively closed (by Lemma 2$), \phi \in G\left(\left(A_{i}\right)_{i \in N}\right)$, as desired.

Proof of Lemma 11. If (d) is violated, there is a set $S$ that is complete w.r.t. $P$ and s.t. for some $\varphi \in \Phi \backslash P$, both $S \cup\{\varphi\}$ and $S \cup\{\neg \varphi\}$ are consistent. These two sets can be extended, so that there are $B, B^{\prime} \in D$ with $B \cap P=B^{\prime} \cap P$, but $B \neq B^{\prime}$. Let 1 be any individual in $N$, and let $F: D^{N} \rightarrow D$ be defined by the condition that for all $\left(A_{i}\right)_{i \in N} \in D^{N}$,

$$
A= \begin{cases}B & \text { if } A_{1}=B^{\prime} \text { and } A_{i}=B \text { for all } i \in N \backslash\{1\} \\ A_{1} & \text { otherwise. }\end{cases}
$$


This $F$ is not dictatorial, and it is systematic, hence independent, as well as unanimitypreserving on $P$, because 1 is a dictator on $P$.

Proof of Proposition 1. Let $F$ be a filter rule with filter $\mathcal{C}$. Take $\left(A_{i}\right)_{i \in N} \in D^{N}$ and consider $A$. We first prove that $A$ is consistent. If not, (L6) implies that $A$ has a finite minimal inconsistent subset $Y$. We have $N_{p} \in \mathcal{C}$ for all $p \in A$, hence for all $p \in Y$. From properties of a filter, $\cap_{p \in Y} N_{p} \in \mathcal{C}$, so $\cap_{p \in Y} N_{p} \neq \emptyset$. Now, an individual $j \in \cap_{p \in Y} N_{p}$ is s.t. $Y \subseteq A_{j}$, which contradicts the consistency of JS in $D$.

We now show that $A$ is deductively closed. Take any $q \in \Phi$ with $A \vdash q$. Among all subsets of $A$ entailing $q$, there is a finite minimal one $Z$ by (L6). By the same argument as before, $N_{p} \in \mathcal{C}$ for all $p \in Z$, and $\emptyset \neq \cap_{p \in Z} N_{p} \in \mathcal{C}$. All $i \in \cap_{p \in Z} N_{p}$ are s.t. $Z \subseteq A_{i}$, and hence s.t. $q \in A_{i}$ because JS in $D$ are deductively closed. Thus, $\cap_{p \in Z} N_{p} \subseteq N_{q}$, which implies that $N_{q} \in \mathcal{C}$, and hence that $q \in A$.

If $F$ is an ultrafilter rule, its images are consistent from what has been shown, and complete, as one can check using the fact that individual JS in $D$ are complete.

Proof of Proposition 2. Let $N$ be infinite. Clearly, the dictatorial $F$ are the ultrafilter rules $F: D^{N} \rightarrow 2^{\Phi}$ generated by ultrafilters $\mathcal{C}$ that are principal, i.e., s.t. $\mathcal{C}=\{C \subseteq N:\{i\} \subseteq C\}$ for some $i \in N$. It is known that ultrafilters $\mathcal{C}$ on an infinite $N$ are either principal or free, i.e., s.t. $\cap_{C \in \mathcal{C}} C=\emptyset$. Now, we show that free ultrafilters generate finitely anonymous $F$. Observe first that if $\mathcal{C}$ is free, $\mathcal{C}$ contains no finite set. For, if $E=\left\{i_{1}, \ldots, i_{k}\right\} \in \mathcal{C}$, then (we leave this for the reader to check) there is $i_{j}$ s.t. $\left\{i_{j}\right\} \subseteq \mathcal{C}$ and $\mathcal{C}$ is principal. Second, this observation implies that for whatever $C \in \mathcal{C}, C \backslash Z \in \mathcal{C}$ if $Z \subseteq N$ is finite (we leave this again to the reader).

Now, let $F$ be a SJF generated by a free ultrafilter and $\pi: N \rightarrow N$ a finite permutation, and for any $\left(A_{i}\right)_{i \in N} \in D^{N}$, compare $A=F\left(\left(A_{i}\right)_{i \in N}\right)$ and $A^{\prime}=F\left(\left(A_{\pi(i)}\right)_{i \in N}\right)$. By Proposition $1, A, A^{\prime} \in D$. If we prove that $A \subseteq A^{\prime}$, we will have proved that $A=A^{\prime}$ because the completeness and consistency of these JS makes $A \mp A^{\prime}$ an impossibility. Let $\phi \in A$; then, $N_{\phi} \in \mathcal{C}$. Because $Z=\{i: \pi(i) \neq i\}$ is finite, the last observation implies that $N_{\phi} \backslash Z \in \mathcal{C}$. Hence, as a superset, $\left\{i: \phi \in A_{\pi(i)}\right\} \in \mathcal{C}$, which implies that $\phi \in A^{\prime}$, as desired.

It remains to exhibit a free ultrafilter. The classic example takes the collection of all subsets of $N$ with finite complementaries. This is clearly a filter, and as any filter, it can be extended to an ultrafilter by using Zorn's lemma (for a detailed proof, see Chang and Keisler, 1973, p. 167). The resulting ultrafilter is free; for otherwise it would be principal, hence with singleton (rather than empty) intersection.

Proof of Proposition 3. Consider $N$ and $\kappa$ as specified. If $\kappa$ is finite, the claim follows from the possibility result on Finite Anonymity remarked in the main text. If $\kappa$ is infinite, consider the set of coalitions with complementaries of cardinality at most $\kappa$. This is a filter, which can be extended to an ultrafilter $\mathcal{F}$. Since $\kappa<|N|, \mathcal{F}$ can only contain sets of cardinality greater than than $\kappa$, and since $\mathcal{F}$ is either principal or free, it is free. Now, if $C \in \mathcal{F}$, and $Z \subseteq N$ has cardinality at most $\kappa$, then $C \backslash Z \in \mathcal{F}$ (by a more general form of an observation made in the proof of Proposition 2). That the SJF generated by $F$ is anonymous up to $\kappa$ follows from the second paragraph in the proof of Proposition 2. 


\section{References}

Chang, C.C., Keisler H.J. (1973) Model Theory, Amsterdam, Elsevier.

Dietrich, F. (2006) Judgment Aggregation: (Im)Possibility Theorems, Journal of Economic Theory 126(1): 286-298

Dietrich, F. (2007) A Generalized Model of Judgment Aggregation, Social Choice and Welfare 28(4): 529-565

Dietrich, F (forthcoming) The Possibility of Judgment Aggregation on Agendas with Subjunctive Implications, Journal of Economic Theory.

Dietrich, F., List, C. (2005) The Impossibility of Unbiased Judgment Aggregation, WP, The London School of Economics

Dietrich, F., List, C. (2007) Arrow's Theorem in Judgment Aggregation, Social Choice and Welfare 29(1): 19-33

Dietrich, F., List, C. (forthcoming) Judgment Aggregation Without Full Rationality, Social Choice and Welfare

Dokow, E., Holzman, R. (forthcoming) Aggregation of Binary Evaluations, Journal of Economic Theory

Dokow, E., Holzman, R. (2006) Aggregation of Binary Evaluations with Abstentions, WP, Technion Israel Institute of Technology

Dokow, E., Holzman, R. (2007) Aggregation of Binary Evaluations for Truthfunctional Agendas, WP, Technion Israel Institute of Technology

Fishburn, P. (1970) Arrow's Impossibility Theorem: Concise Proof and Infinite Voters, Journal of Economic Theory, 2: 103-106.

Gärdenfors, P. (2006) An Arrow-like Theorem for Voting with Logical Consequences, Economics and Philosophy 22(2): 181-190.

Heifetz, A., Mongin D. (2001) Probability Logic for Type Spaces, Games and Economic Behavior, 35:31-53.

Kirman, A., Sonderman D. (1972), Arrow's Theorem, Many Agents, and Invisible Dictators, Journal of Economic Theory, 5, 267-277.

Lauwers, L. (1998) Intertemporal Objective Functions: Strong Pareto versus Time Neutrality, Mathematical Social Sciences 35: 37-55

Lauwers, L., van Liedekerke, L. (1995) Ultraproducts and Aggregation, Journal of Mathematical Economics 24: 217-237

Lismont, L., Mongin P. (2003) Strong Completeness for Weak Logics of Common Belief, Journal of Philosophical Logic, 32: 115-137.

List, C., Pettit, P. (2002) Aggregating Sets of Judgments: An Impossibility Result, Economics and Philosophy 18(1): 89-110

Mongin, P. (forthcoming) Factoring Out the Impossibility of Logical Aggregation, Journal of Economic Theory.

Nehring, K. (2006) Oligarchies in Judgment Aggregation, WP, University of California at Davies

Nehring, K., Puppe, C. (2002) Strategy-Proof Social Choice on Single-Peaked Domains: Possibility, Impossibility and the Space Between, WP, University of California 
at Davies

Nehring, K., Puppe, C. (forthcoming) Consistent Judgement Aggregation: The Truth-Functional Case, Social Choice and Welfare.

Nehring, K., Puppe, C. (2007) Justifiable Group Choice, WP, Karlsruhe University

Pauly, M., van Hees, M. (2006) Logical Constraints on Judgment Aggregation, Journal of Philosophical Logic 35(6): 569-585

van Hees, M. (2007) The Limits of Epistemic Democracy, Social Choice and Welfare 28(4): 649-666

Vieille, N. (2007), When is Coherent Aggregation Possible?, WP, HEC Business School. 